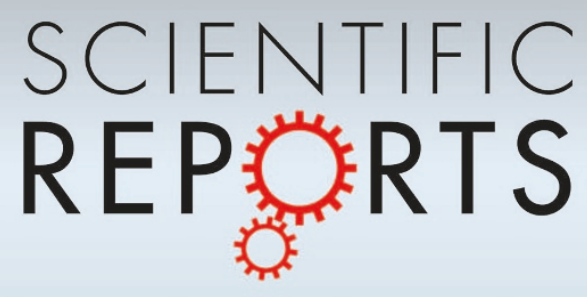

\title{
OPEN Deleterious effect of suboptimal diet on rest-activity cycle in Anastrepha ludens manifests itself with age \\ SUBJECT AREAS: AGEING
}

EVOLUTION

ECOLOGY

BEHAVIOURAL ECOLOGY

Received

10 November 2012

Accepted

15 April 2013

Published

3 May 2013

Correspondence and requests for materials should be addressed to

J.R.C. (jrcarey@ ucdavis.edu)
Joanna C. Chiv', Kevin Kaub', Sige Zou², Pablo Liedo ${ }^{3}$, Leopoldo Altamirano-Robles ${ }^{4}$, Donald Ingram ${ }^{5}$ \& James R. Carey'

'Department of Entomology, College of Agricultural and Environmental Sciences, University of California, Davis, CA 95616

${ }^{2}$ Translational Gerontology Branch, National Institute on Aging, Baltimore, MD, ${ }^{3}$ Departamento de Entomologia, El Colegio de la Frontera Sur (ECOSUR), Tapachula, Chiapas, Mexico, ${ }^{4}$ Laboratorio de Vision por Computadora, Instituto Nacional de Astrofisica Optica y Electronica (INAOE), Tonantzintla, Puebla, Mexico, ${ }^{5}$ Nutritional Neuroscience and Aging Laboratory, Pennington Biomedical Research Center, Louisana State University, Baton Rouge, LA.

Activity patterns and sleep-wake cycles are among the physiological processes that change most prominently as animals age, and are often good indicators of healthspan. In this study, we used the video-based high-resolution Behavioral Monitoring System (BMS) to monitor the daily activity cycle of tephritid fruit flies Anastrepha ludens over their lifetime. Surprisingly, there was no dramatic change in activity profile with respect to age if flies were consistently fed with a nutritionally balanced diet. However, if flies were fed with sugar-only diet, their activity profile decreased in amplitude at old age, suggesting that suboptimal diet affected activity patterns, and its detrimental effect may not manifest itself until the animal ages. Moreover, by simulating different modes of behavior monitoring with a range of resolution and comparing the resulting conclusions, we confirmed the superior performance of video-based monitoring using high-resolution BMS in accurately representing activity patterns in an insect model.

ging in animals leads to changes in physiology and metabolism, which manifests into characteristic changes in behavior ${ }^{1,2}$. In healthy humans, aging is associated with decreases in both sleep quantity and quality as measured by the number of awakenings after the onset of the sleep period ${ }^{3}$. In the fruit fly Drosophila melanogaster, which has become a powerful model organism for the study and mechanistic dissection of sleep in recent years ${ }^{4-13}$, sleep-wake cycle also appears to break down with age to varying degree depending on genetic background and rearing conditions ${ }^{14,15}$. As opposed to the decrease in total sleep amount that accompanies the aging process in humans, wild type D. melanogaster shows an increase in sleep quantity as the animal ages ${ }^{4}$. Age-dependent changes of sleep can partly be attributed to the weakening of endogenous circadian rhythms ${ }^{15}$. Sleep-wake cycle has been widely used as an output parameter for assessing defects in circadian rhythms ${ }^{16-18}$. In laboratory conditions with constant temperature and abrupt change of lighting conditions between day to night or night to day transitions, $D$. melanogaster generally exhibits two bouts of activity; one centered around ZT0 (ZT = Zeitgeiber time; equivalent to dawn) termed morning peak and another around ZT12 (equivalent to dusk) termed evening peak. These two bouts of activities are controlled by the endogenous circadian clock, and can be observed even in free-running conditions, such as total darkness, once the endogenous clock has been entrained to a certain environmental regime. Studies in the molecular mechanisms of sleep as well as circadian rhythm control and regulation in D. melanogaster, and more recently in other invertebrates such as the malaria vector mosquito Anopheles gambiae ${ }^{19}$, have relied on the use of DAMS (Drosophila Activity Monitoring System) (Trikinetics, Waltham, MA). However, several studies showed that perhaps a video-based system may give a more accurate approximation of sleep-wake cycle and provide a finer resolution necessary to assess certain parameters of sleep, for example sleep quality and fragmentation ${ }^{20-25}$. More importantly, videobased recording systems have the superior capability of longer recording time due to the flexibility of how the animal habitats can be set up. This is important especially when monitoring sleep-wake cycle and other behavior that might change as animal ages.

The main objective of this study was to use the video-based high-resolution Behavioral Monitoring System $(\mathrm{BMS})^{26}$ to monitor the daily rest-activity cycle of tephritid Mexican fruit flies Anastrepha ludens over their lifetime, and to determine how this behavioral parameter changes with respect to diet and age. Note that without 
empirical evidence that the inactivity in Anastrepha ludens we are observing in our experiments represents bona-fide sleep as defined in D. melanogaster and mammals, e.g. whether it is accompanied by changes in arousal threshold ${ }^{11}$, we referred the inactivity we are assessing in our study as rest instead of sleep. Clinical studies using human subjects indicate that food can affect the quality of sleep, and dietary choice can be influenced by the quantity of sleep ${ }^{27}$. A. ludens has a relatively long lifespan as compared to other insect models with adults living for 3 to 4 months on average and up to 11 months in the wild, and has been previously used in demographic studies to study aging and aging intervention ${ }^{28-31}$, therefore representing a good model for our studies. Moreover, A. ludens is regarded as a very serious insect pest of various fruits, especially citrus crops in Mexico and other parts of Central America ${ }^{32,33}$. Continual monitoring and eradication is necessary to prevent its invasion into the United States, and understanding the basic physiology of this insect pest will also benefit the design and implementation of control strategies. A secondary objective of this study was to determine the minimum monitoring resolution that will allow the accurate representation of daily activity pattern in this insect model. This was achieved by simulating different modes of behavior monitoring, i.e. enclosure bisection and distance displacement, using the high-resolution data recorded using BMS, and comparing the conclusions based on behavior monitoring at different resolution.

\section{Results}

Models of behavioral monitoring. BMS was developed as the first fully automated video system capable of capturing, and more importantly classifying lifetime behaviors of an invertebrate model,
A. ludens $^{26}$. In addition to differentiating between behaviors such as walking, resting, flying, feeding, drinking, and micro-movements, BMS also records the 3D location of individual animals, allowing researchers to collect high resolution data concerning the effects of age, diet, genetics, and any experimental manipulations on behavior, mobility, and general health conditions of animals. In this study, we used BMS to examine the rest-activity pattern of A. ludens, and determine how it changed with age as well as dietary conditions. With the high-resolution data obtained using BMS, we have the opportunity to systematically determine the minimum recording resolution necessary to most accurately represent rest-activity pattern in this invertebrate model. High-resolution behavioral data was recorded using BMS throughout the lifetime of 32 female animals, and their daily activity patterns were calculated based on three different models of criteria (Figure 1). The enclosure bisection model provides the lowest resolution and is most similar to the activity-monitoring concept of DAMS (Trikinetics, Waltham, MA) (Figure 1A). DAMS is based on using the interruption of an infrared light beam to record the locomotor activity of individual animals contained inside small tubes. When measured over many days, animals exhibit daily cycles of activity and inactivity ${ }^{18}$. To analyze our data using the enclosure bisection simulation, we bisected the BMS enclosure in both the $\mathrm{X}$ - and $\mathrm{Y}$-axis to create an imaginary "infrared beam". Only one of the two possible bisections (X- or Yaxis) is shown in Fig. 1A to illustrate the concept. The fly was only considered active when it "broke the beam". Note that if the movement of the fly was confined to either side of the "beam", it was considered as inactive or resting, therefore this model is most likely to underestimate activity levels. Although the enclosure
A

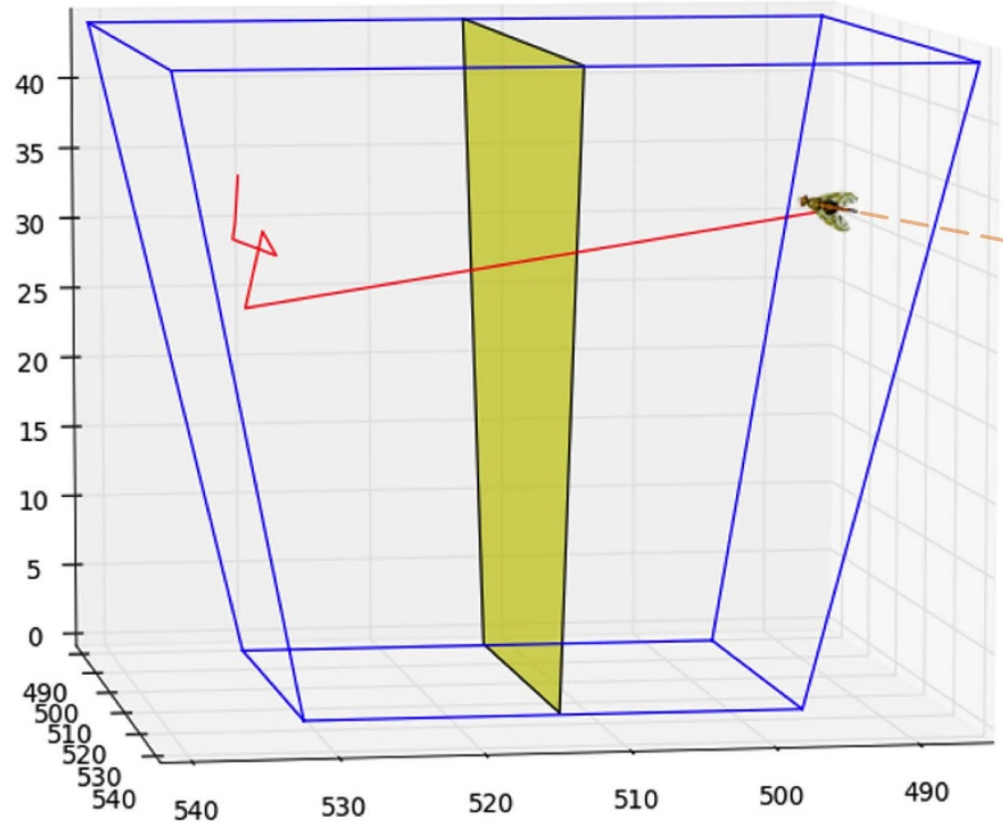

B

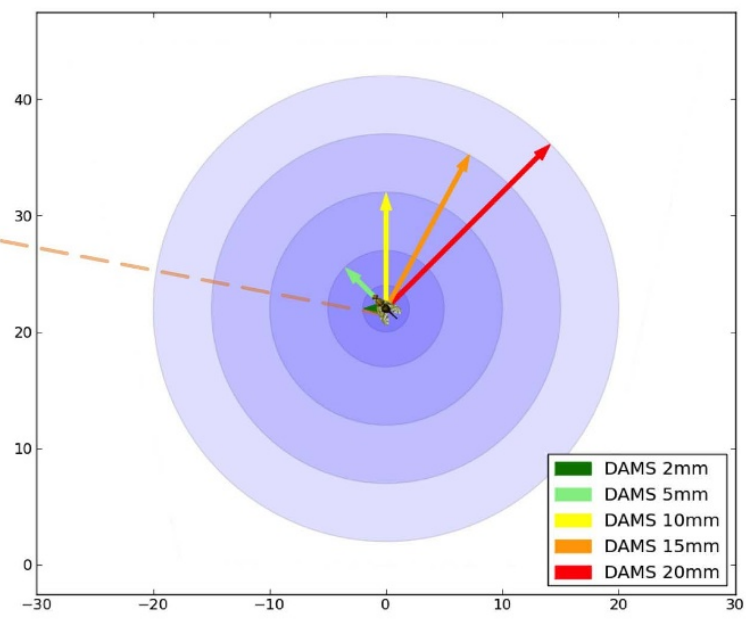

Figure 1 Schematic diagrams illustrating the different models for measuring and interpreting activity of Anastrepha ludens: BMS, simulated distance displacement models, and simulated enclosure bisection models. The fly visible in the diagrams represents Anastrepha ludens: a body at 3 mm in length in an enclosure (cage) measuring $45 \mathrm{~mm}$ high, $35 \times 35 \mathrm{~mm}$ square at the base and $55 \times 55 \mathrm{~mm}$ square at the top. Distances are expressed in millimeter. The red line extending from the fly indicates its path of movement over a 1-minute period. (A) Spatial graphic depicting BMS cage simulating the enclosure bisection model; the fly enclosure is bisected by a virtual vertical plane (shaded yellow). Only one axis of bisection (X or Y) is shown here. The bisection model resembles the activity detection concept in traditional DAMS, where an activity event is recorded only when a fly crosses the yellow plane (i.e. breaks the infrared beam of the DAMS). (B) BMS cage simulating a displacement distance concept; each circle/sphere represents a different displacement distance from the fly's current location in the cage. (Red, orange, yellow, light green, and dark green represent displacement distances of $20 \mathrm{~mm}, 15 \mathrm{~mm}, 10 \mathrm{~mm}, 5 \mathrm{~mm}$, and $2 \mathrm{~mm}$ respectively.) DDS = Distance Displacement Simulation. An activity event is recorded when a fly moves beyond the given displacement distance and the fly's new location becomes its new current location. The BMS system provides the highest resolution recording including behavior characterized as micro-movements, which has a displacement distance of less than $2 \mathrm{~mm}$. 
bisection model bears the closest resemblance to the DAMS in concept, it is important to point out that DAMS enclosures are usually narrow tubes, where the movement of an animal is restricted to a single dimension. As a result, the animal is more likely to break the infrared beam when they are active, therefore partially compensating for the underestimation of movement occurring on either side of the beam. On the other hand, the animal is not restricted to movement in one dimension in the 3D BMS enclosures used in our experiments (Fig. 1A). This will most likely lead to an increase in activity that does not "break the beam" as compared to recordings using traditional DAMS, and exaggerate the underestimation of activity as deduced by the enclosure bisection simulation. For this reason, the goal of our simulation study is not to make a direct comparison between the DAMS and BMS, which is not justified due to the difference in enclosure dimension used, but rather, to determine the minimum recording resolution in BMS that most accurately approximates actual activity patterns by comparing results obtained using the enclosure bisection simulation, distance displacement simulation, to actual results as measured by BMS.

The second model, which we predict would increase the resolution of activity vs. inactivity, is the distance displacement model (Figure 1B). Here, a fly was considered to be active once it crossed a threshold distance. A number of threshold distances $(2 \mathrm{~mm}, 5 \mathrm{~mm}$, $10 \mathrm{~mm}, 15 \mathrm{~mm}, 20 \mathrm{~mm}$ ) were implemented during our simulation to assess the minimum distance necessary to approximate the differentiation of active vs. inactive as determined by BMS, which we considered to be the most accurate representation of actual activity patterns.

Analysis of $A$. ludens activity patterns with respect to age and diet. Based on the different criteria for active vs. inactive or rest, we calculated the total rest amount (expressed as rest intensity) for flies during the 12-hour light period vs. 12-hour dark period during each circadian day, and plotted the data as event history charts over the lifetime of the flies (Figure 2). These charts are useful in depicting general trends over the lifetime of experimental animals. As clearly illustrated by the color scheme (green = less rest; red = more rest), the amount of rest in the light period, as recorded using BMS and illustrated in the bottom row of each chart, is lower than in the dark period throughout the lifetime of the flies in both dietary conditions (compare top panels of Figure 2A and B; and bottom panels of Figure $2 \mathrm{~A}$ and $\mathrm{B})$. This is in agreement with other video-based assays performed in $D$. melanogaster, suggesting flies have a similar sleep-wake pattern as in humans, where sleep is consolidated in the dark phase (e.g. Ref. 22). The average rest intensity in the light period

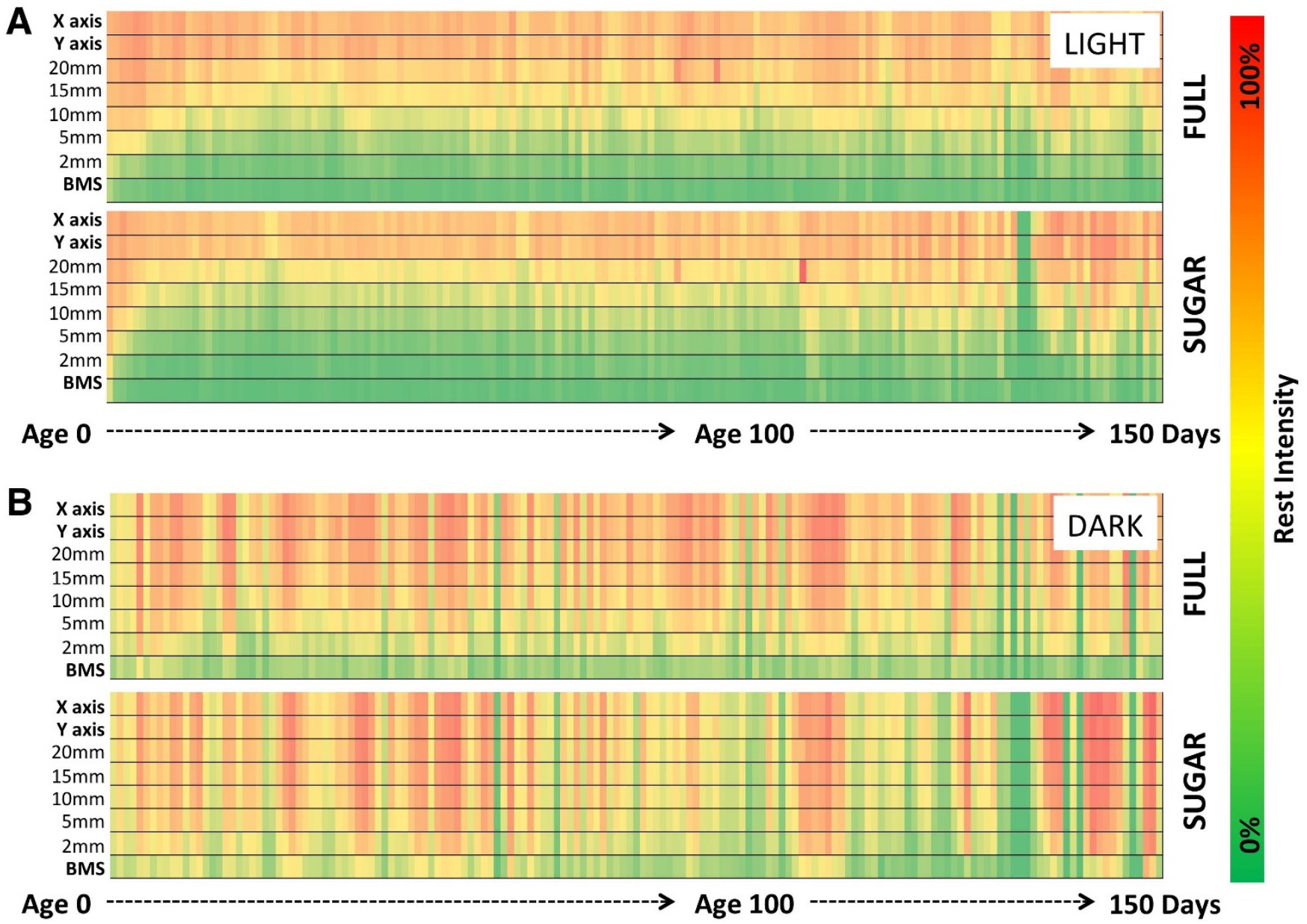

Figure $2 \mid$ Event history charts depicting the average daily rest intensity over lifetime of Anastrepha ludens in light and dark phases of the day as compared using BMS, displacement distance simulation, and enclosure bisection simulation. Rest intensity is calculated as the percentage of rest-time over the (A) light or (B) dark period of a given day, $0 \%$ being active and 100\% being 12 hours of continuous rest. Columns represent rest intensity for a specified 12-hour period for a specific age; age increases one day per column from left to right on the X-axis of the graphs. Rows depict rest intensity as calculated by different models: From top to bottom on the Y-axis of the graphs, enclosure bisection simulation: X-axis, Y-axis, distance displacement simulation: $20 \mathrm{~mm}, 15 \mathrm{~mm}, 10 \mathrm{~mm}, 5 \mathrm{~mm}, 2 \mathrm{~mm}$, and BMS respectively. Data is presented for the Light phase (12-hour lights-on periods), Dark phase (12-hour lights-off periods) for female animals on Full $(\mathrm{n}=16)$ or Sugar $(\mathrm{n}=16)$ diet. 
did not seem to differ dramatically between flies that were fed with full vs. sugar diet upon visual inspection of the BMS event history charts (Figure 2A), even though the differences were significant $(\mathrm{p}<0.0066)$ (Figure 3A). On the other hand, the average sleep intensity in the dark period between flies fed with the different diets showed clearly observable and highly significant differences $\left(\mathrm{p}<4.76^{\mathrm{E}-09}\right.$ ) (Figure $2 \mathrm{~B}$ and $3 \mathrm{~A}$ ), suggesting that sugar-only diet resulted in an increase in rest intensity at "night". In comparing the different simulation models for calling "activity" vs. "rest" to assessment by BMS, the amount of rest was greatly overestimated in the light period irrespective of diet, especially in those models with resolution coarser than $5 \mathrm{~mm}$ (Figure 2A). The degree of rest overestimation was not as severe during the dark period (Figure 2B), but still presented significant differences (Figure 3B).

To compare age-dependent differences more carefully, we examined activity patterns of flies at different stages of their life: young flies (15 to 25-day old), middle-aged flies (50 to 60-day old), and old flies (80 to 90-day old) (Figure 4). Based on the BMS-deduced data (shown in red), feeding flies with a sugar-only diet led a decrease in day-time rest (compare Figure $4 \mathrm{~A}$ and $\mathrm{C}$ ) and an increase in nighttime rest (compare Figure 4B and D) as compared to feeding flies with full diet. This observation is consistent in all age groups. It is interesting to note that flies that were on a sugar-fed diet had a shorter lifespan ${ }^{26}$. Although flies fed with sugar-only diet showed an increase in dark-period rest throughout all age groups, it is unclear whether the quality and restorative function of their rest is equivalent to flies fed with full-balanced diet. Together with other physiological consequences of sugar-only diet, this may have long-term cumulative detrimental effects on their healthspan, which manifested into lower nighttime rest intensity at old age (Figure 4D) and shorter lifespan. In D. melanogaster, it was reported that high calorie diet, which is associated with acceleration in the aging process and reduction in lifespan across species, also accelerates age-associated rest changes ${ }^{34}$.

Finally, we examined the daily activity rhythms of flies across three different age groups (Figures 5 and 6). These results were based on average activity rhythms over an 11-day period for each age group. BMS recordings indicated that $A$. ludens increases their activity levels at 'dawn', the beginning of the light period. As opposed to other species such as D. melanogaster, for which daily activity patterns have been studied extensively, there is virtually no increase in activity levels in anticipation for dawn. This may be indicative of a weak "morning oscillator" 35,36 in A. ludens, which is predicted to control the timing of morning activity. Or perhaps, A. ludens relies more on temperature increase as a cue to anticipate dawn. Temperature has been shown to be a critical variable in controlling daily activity patterns ${ }^{37}$, and may even be dominant over light conditions in natural environment ${ }^{38}$. It will be interesting to observe activity patterns for $A$. ludens in conditions that more closely resemble natural transitions between day to night as well as night to day, which are richer in environmental cues. A. ludens flies remain active throughout the day and their activity reach maximum levels at 'dusk', just before the beginning of the dark period. This peak of activity is coincident with or is preceded by the peak of mating activity ${ }^{39}$. This is followed by a rapid decrease in activity levels, resulting in consolidated 'rest' throughout the dark period. In comparing flies fed with full diet, sugar-fed flies have higher average as well as peak activity levels in

\begin{tabular}{|c|c|c|}
\hline \multicolumn{3}{|c|}{ FULL vs. SUGAR } \\
\hline P-Values & Lights ON & Lights OFF \\
\hline EBS - $X$ & 0.0024 & 0.00047 \\
\hline$E B S-Y$ & 0.0117 & 0.00033 \\
\hline DDS - 20mm & 2.43E-07 & 0.00098 \\
\hline DDS $-15 \mathrm{~mm}$ & 8.77E-18 & 0.0073 \\
\hline DDS - $10 \mathrm{~mm}$ & $8.78 \mathrm{E}-17$ & 0.0796 \\
\hline DDS - $5 \mathrm{~mm}$ & $1.01 \mathrm{E}-11$ & 0.3650 \\
\hline DDS - $2 \mathrm{~mm}$ & $2.26 \mathrm{E}-08$ & 0.0424 \\
\hline BMS & 0.0066 & 4.76E-09 \\
\hline
\end{tabular}

\section{B Lights OFF}

\begin{tabular}{|c|c|c|c|c|c|c|c|c|c|}
\hline \multirow[t]{9}{*}{ FULL } & P-Values & EBS & $\%-Y$ & $\begin{array}{l}\text { DDS - } \\
20 \mathrm{~mm}\end{array}$ & $\begin{array}{l}\text { DDS - } \\
15 \mathrm{~mm}\end{array}$ & $\begin{array}{l}\text { DDS - } \\
10 \mathrm{~mm}\end{array}$ & $\begin{array}{l}\text { DDS - } \\
5 \mathrm{~mm}\end{array}$ & $\begin{array}{l}\text { DDS - } \\
2 \mathrm{~mm}\end{array}$ & BMS \\
\hline & EBS- $X$ & & 0.4313 & 0.1536 & 50.0035 & 3 E- 07 & $1 \mathrm{E}-19$ & $9 \mathrm{E}-40$ & $2 E-74$ \\
\hline & EBS-Y & 0.4313 & & 0.1179 & 0.0022 & 2. $1 \mathrm{E}-07$ & $6 \mathrm{E}-20$ & $8 \mathrm{E}-40$ & $8 E-74$ \\
\hline & DDS - $20 \mathrm{~mm}$ & 0.1536 & 0.1179 & & 0.0475 & $3 \mathrm{E}-05$ & $4 \mathrm{E}-16$ & $1 E-35$ & $4 \mathrm{E}-71$ \\
\hline & DDS - $15 \mathrm{~mm}$ & 0.0035 & 0.0022 & 0.0475 & & 0.0091 & 2E-11 & $4 E-30$ & $3 E-68$ \\
\hline & $\mathrm{DDS}-10 \mathrm{~mm}$ & $3 E-07$ & $1 \mathrm{E}-07$ & $3 E-05$ & 0.0091 & & $4 \mathrm{E}-06$ & $2 \mathrm{E}-22$ & $9 E-64$ \\
\hline & DDS - $5 \mathrm{~mm}$ & $1 \mathrm{E}-19$ & $6 \mathrm{E}-20$ & $4 E-16$ & $52 \mathrm{E}-11$ & LE-06 & & $1 \mathrm{E}-09$ & $3 E-53$ \\
\hline & $\mathrm{DDS}-2 \mathrm{~mm}$ & $9 E-40$ & $8 \mathrm{E}-40$ & $1 E-35$ & $4 E-30$ & $2 E-22$ & $1 \mathrm{E}-09$ & & $3 E-33$ \\
\hline & BMS & $2 E-74$ & $8 \mathrm{E}-74$ & $4 \mathrm{E}-71$ & 1 $3 E-68$ & 3) $9 \mathrm{E}-64$ & $3 E-53$ & $3 E-33$ & \\
\hline \multirow[t]{9}{*}{ SUGAR } & P-Values & EBS & & $\begin{array}{l}\text { DDS - } \\
20 \mathrm{~mm}\end{array}$ & $\begin{array}{l}\text { DDS - } \\
15 \mathrm{~mm}\end{array}$ & $\begin{array}{l}\text { DDS - } \\
10 \mathrm{~mm}\end{array}$ & $\begin{array}{l}\text { DDS - } \\
5 \mathrm{~mm}\end{array}$ & $\begin{array}{l}\text { DDS - } \\
2 \mathrm{~mm}\end{array}$ & BMS \\
\hline & EBS $-X$ & & 0.4882 & 0.2572 & 20.0946 & 50.0163 & $6 \mathrm{E}-05$ & $8 \mathrm{E}-12$ & $3 E-31$ \\
\hline & EBS $-Y$ & 0.4882 & & 0.2663 & 0.0991 & 0.0173 & $6 \mathrm{E}-05$ & $9 \mathrm{E}-12$ & $3 E-31$ \\
\hline & DDS - $20 \mathrm{~mm}$ & 0.2572 & 0.2663 & & 0.2531 & 0.0678 & 0.0006 & $4 \mathrm{E}-10$ & $4 E-29$ \\
\hline & DDS - $15 \mathrm{~mm}$ & 0.0946 & 0.0991 & 0.2531 & & 0.2043 & 0.005 & $2 \mathrm{E}-08$ & $8 \mathrm{E}-27$ \\
\hline & DDS $-10 \mathrm{~mm}$ & 0.0163 & 0.0173 & 0.0678 & 30.2043 & & 0.039 & $9 \mathrm{E}-07$ & $3 E-24$ \\
\hline & DDS $-5 \mathrm{~mm}$ & $6 \mathrm{E}-05$ & $6 \mathrm{E}-05$ & 0.0006 & 0.005 & 0.039 & & 0.001 & $1 \mathrm{E}-18$ \\
\hline & DDS - $2 \mathrm{~mm}$ & $8 \mathrm{E}-12$ & $9 \mathrm{E}-12$ & $4 E-10$ & $2 \mathrm{E}-08$ & $39 \mathrm{E}-07$ & 0.001 & & $8 \mathrm{E}-10$ \\
\hline & BMS & $3 E-31$ & $3 E-31$ & $4 E-29$ & $8 \mathrm{E}-27$ & $7 \mathrm{E}-24$ & $1 \mathrm{E}-18$ & $8 \mathrm{E}-10$ & \\
\hline
\end{tabular}

\section{Lights ON}

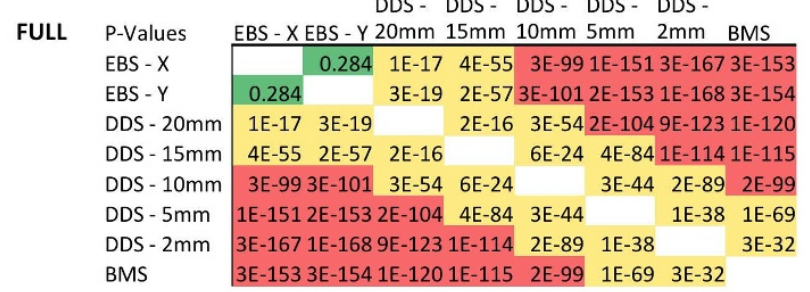

DDS - DDS - DDS - DDS - DDS SUGAR P-Values EBS-XEBS-Y $20 \mathrm{~mm} 15 \mathrm{~mm} 10 \mathrm{~mm} 5 \mathrm{~mm} \quad 2 \mathrm{~mm}$ BMS

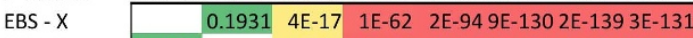

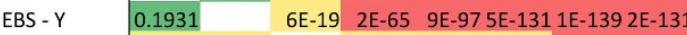
DDS-20mm $4 \mathrm{E}-17 \quad 6 \mathrm{E}-19 \quad 3 \mathrm{E}-10 \quad 1 \mathrm{E}-27 \quad 2 \mathrm{E}-50 \quad 8 \mathrm{E}-63 \quad 9 \mathrm{E}-67$ DDS- $15 \mathrm{~mm} \quad 1 \mathrm{E}-62 \quad 2 \mathrm{E}-65 \quad 3 \mathrm{E}-10 \quad 5 \mathrm{E}-14 \quad 9 \mathrm{E}-50 \quad 1 \mathrm{E}-75 \quad 6 \mathrm{E}-81$ DDS- $10 \mathrm{~mm} \quad 2 \mathrm{E}-94 \quad 9 \mathrm{E}-97 \quad 1 \mathrm{E}-27 \quad 5 \mathrm{E}-14 \quad 1 \mathrm{E}-20 \quad 9 \mathrm{E}-50 \quad 9 \mathrm{E}-61$

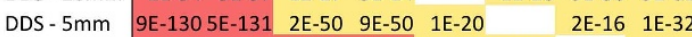

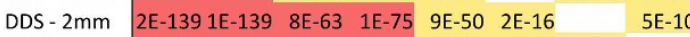

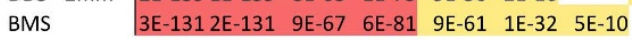

Figure 3 Significance measures between different activity monitoring models. P-values were calculated using the "t-test: Two-Sample Assuming Unequal Variances" from the Data Analysis package in MS Excel. The raw data from Figure 2 was used to generate these P-values. The boxes are shaded in a continuum such that red indicates high levels of significant difference, green indicates no significant differences, and yellow is intermediate. EBS = Enclosure Bisection Simulation; DDS = Distance Displacement Simulation; BMS = Behavioral Monitoring System. 
A
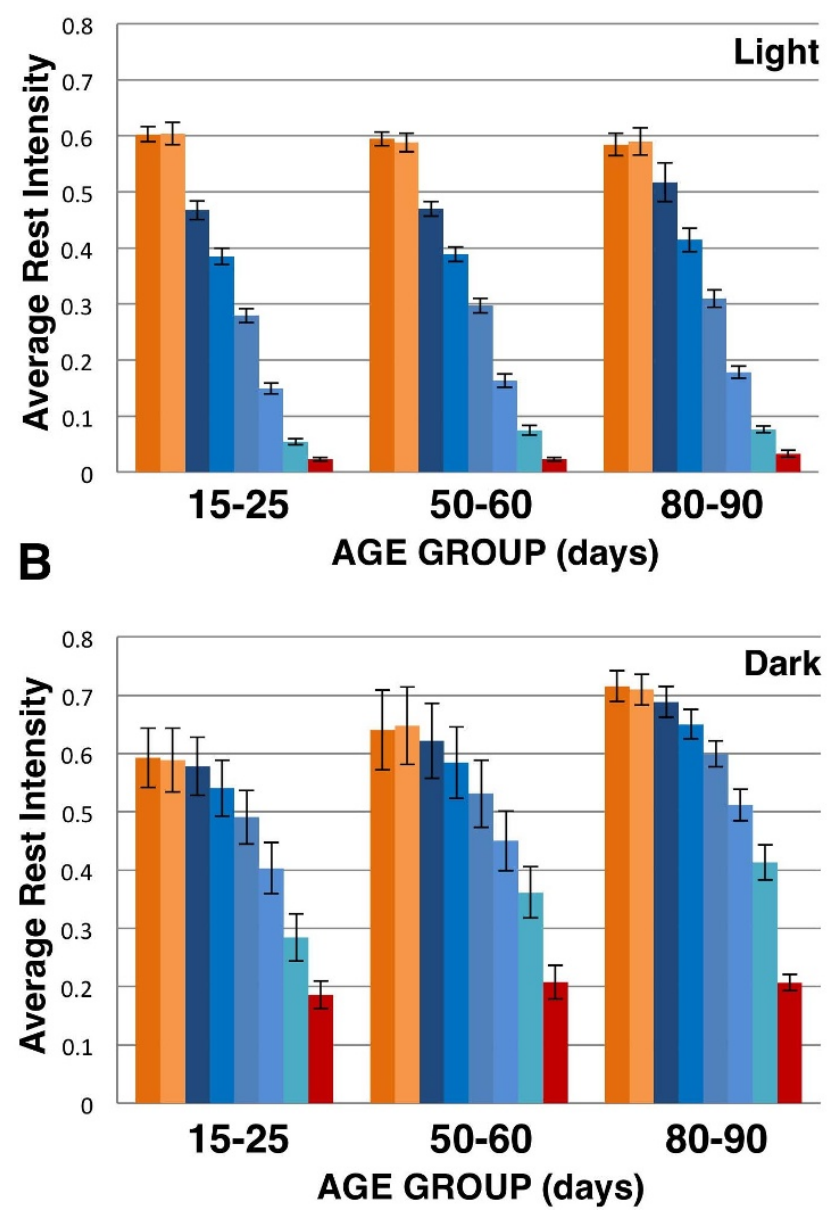

C

Sugar Diet
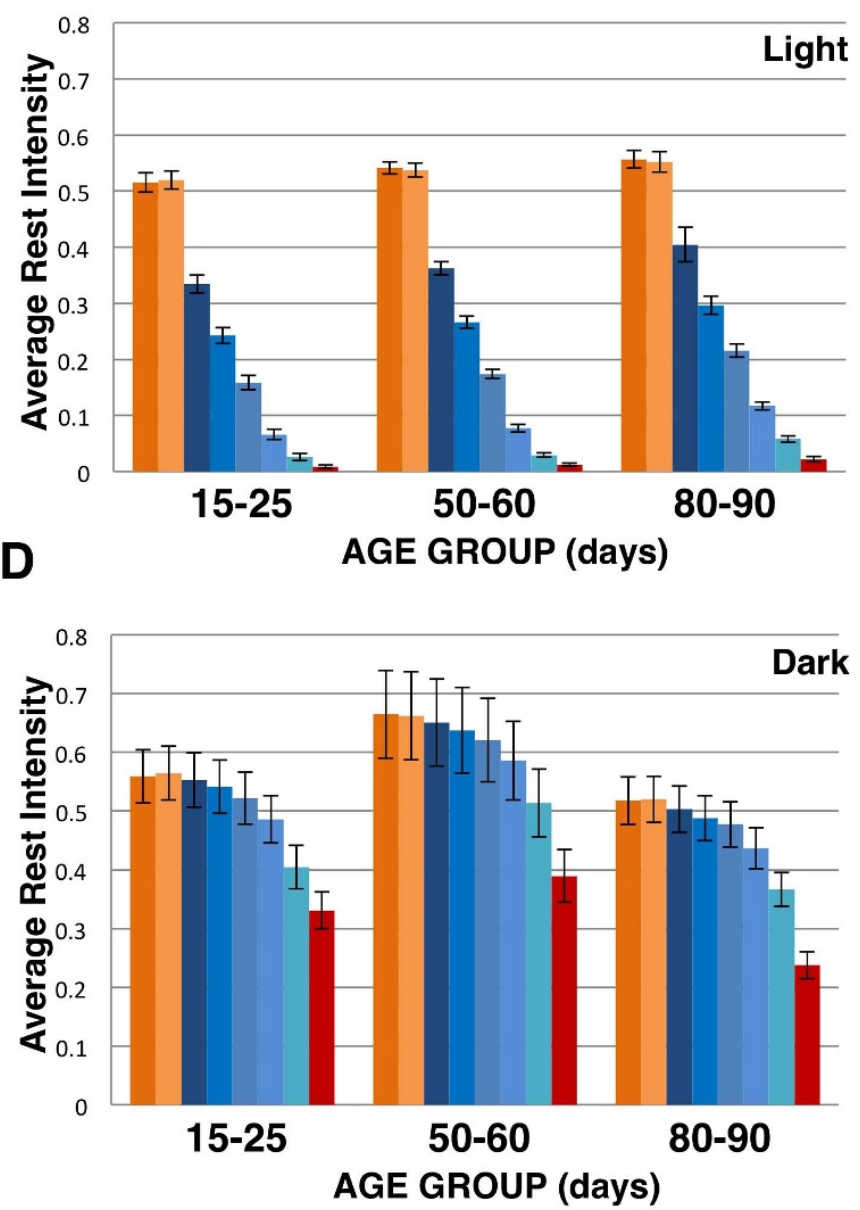

Figure $4 \mid$ Average rest intensity by age groups as interpreted by different activity monitoring models. Bar graphs depicting daily averages of rest intensity over an 11-day period as calculated using different models for light periods or dark (lights-off) periods and full diet or sugar diet flies respectively. Each triad of charts compares rest intensity (left to right) across three age periods, 15-25 days, 50-60 days, and 80-90 days. Each bar denotes the average rest intensity recorded by a specific activity model. Rest intensity is calculated as number of hours of rest/12-hour period (light or dark). This is then averaged over an 11-day period for each age group. Dark orange and light orange bars depict results from enclosure bisection simulation models, $\mathrm{X}$-axis bisection and Y-axis bisection respectively. Five blue-shaded bars from navy to cyan (left to right) depict the results from distance displacement simulation models, $20 \mathrm{~mm}, 15 \mathrm{~mm}, 10 \mathrm{~mm}, 5 \mathrm{~mm}$, and $2 \mathrm{~mm}$ models. Red bars denote the rest levels calculated by the BMS. (A) Rest intensity in full diet A. ludens females during daily 12-hour light periods across ages and models. (B) Full diet average rest intensity during daily 12-hour dark periods. (C) and (D) Average rest intensity in sugar-only diet A. ludens females for daily 12 -hour light periods and dark periods respectively. $\mathrm{n}=16$ for each treatment.

general (compare Figure 5A and D, as well as Figure 5B and E), except when flies reach old age (compare Figure $5 \mathrm{C}$ and $\mathrm{F}$ ). The rapid drop in activity levels at dusk seems to occur slightly earlier in sugarfed flies, and this phenomenon is most obvious in older flies (compare Figure 5C and F). In terms of age-dependent changes in activity profiles, the pattern for flies fed with full diet remains pretty constant throughout their lifetime (Figures $5 \mathrm{~A}-\mathrm{C}$ ), whereas more pronounced differences are observed in sugar-fed flies (Figures 5D-F), perhaps reflecting age-dependent deterioration in healthspan and mobility.

Similar to results shown in figures 2 and 4, there is obvious underestimation of activity by the enclosure bisection and distance displacement models as compared to BMS, especially during the light period (Figures 4 and 5). As a matter of fact, the activity levels of $A$. ludens are quite low as compared to species such as D. melanogaster. Without the use of BMS, it will be difficult to decipher the periodicity of activity rhythms, and these animals may mistakenly be labeled as arrhythmic, simply because their activity levels are too low (Figure 5). Plotting the same data in logarithmic scale allowed us to recover more information on activity profiles even for other monitoring simulations (Figure 6). The simulated enclosure bisection and dis- tance displacement models recorded the same activity profiles as BMS, albeit at a much lower amplitude. Although BMS was originally designed to automate high-resolution behavioral analysis ${ }^{26}$, we believe it provides far superior resolution and sensitivity that will be valuable, even for monitoring of rest-activity cycles and activity patterns.

\section{Discussion}

This is the first report to describe age- and diet-dependent modulation of rest-activity cycle in the tephritid fruit fly Anastrepha ludens. As in humans and other insect models, A. ludens shows consolidated rest during the dark period. Since previous studies in fruit flies and other species reported that the strength of the sleepwake (or rest-activity) cycle generally deteriorates as animal ages ${ }^{8}$, we predicted that we might observe a substantial reduction in amplitude of activity patterns as we assayed tephritid fruit flies of increasing age. Surprisingly, there was no dramatic change in activity patterns throughout their lifetime when flies were kept in light-dark cycles and fed with a more nutritionally balanced diet. Even the overall activity level was only slightly diminished, e.g. as observed during 

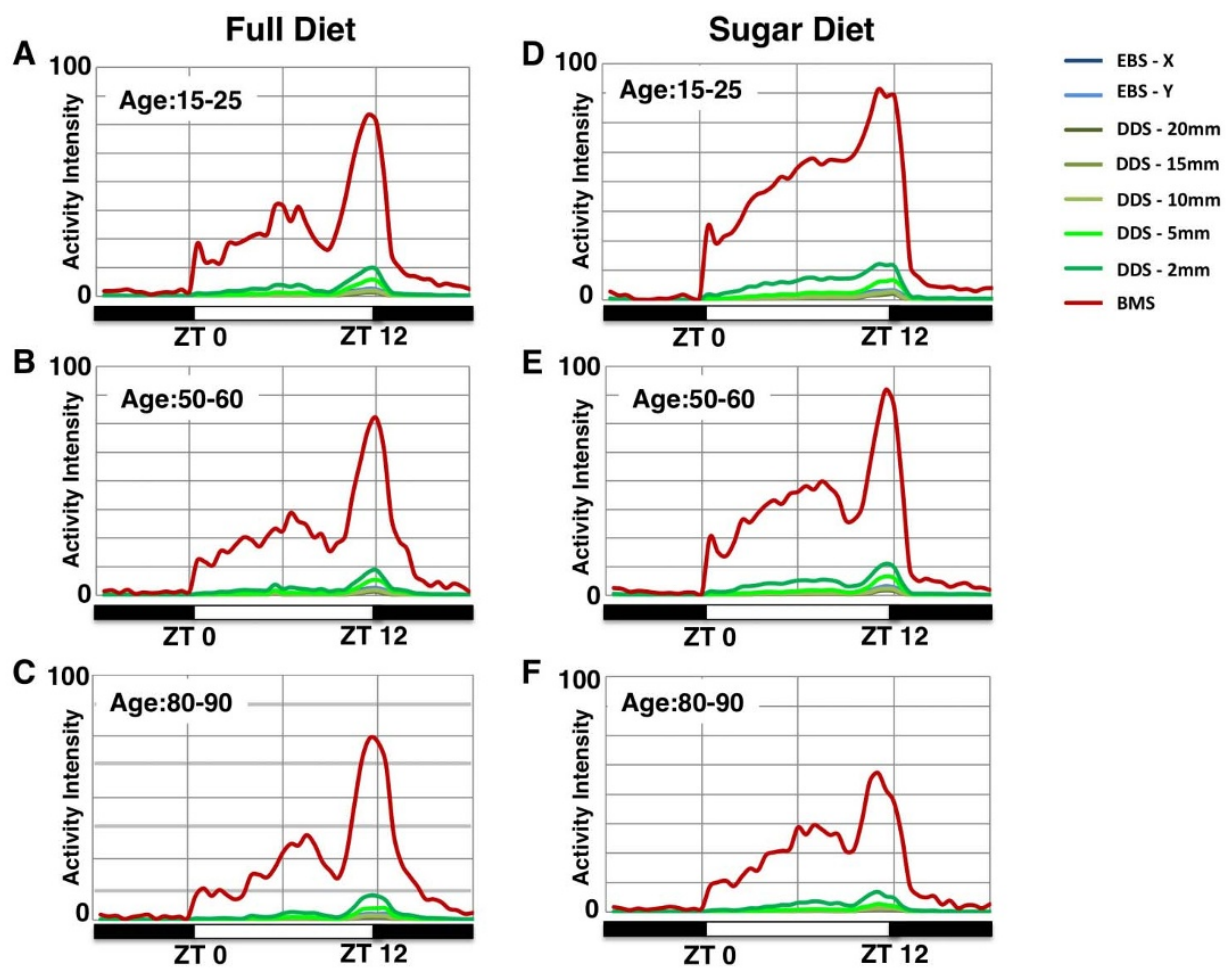

Figure $5 \mid$ Daily activity pattern by age groups as calculated using different activity monitoring models. Average diet-, age-, and model-specific activity patterns of $A$. ludens females through a 24-hour period. Each line represents the diurnal rest-activity patterns of $A$. ludens females averaged over elevenday periods, ages 15-25, 50-60, and 80-90. (A-C) Females reared using full diet; (D-F) Females reared using sugar-only diet ( $\mathrm{n}=16$ ). Activity intensity is calculated for every 30-minute span and expressed as the percentage of time within that 30-minute period when flies are active. This is then averaged over an 11-day period for each of the three age groups. $0 \%$ is no activity, $100 \%$ is continuous activity. Dark bar below chart indicates the daily 12 -hour dark (lights-off) period; white bar indicates the 12-hour light period. ZT0 indicates the time when lights turn on, and ZT12 indicates the time when lights turn off. Results from EBS (enclosure bisection simulation) X- axis and Y-axis bisections are represented by dark and light blue lines. Five green-shaded lines from dark to light depict the results from DDS (distance displacement simulation) $20 \mathrm{~mm}, 15 \mathrm{~mm}, 10 \mathrm{~mm}, 5 \mathrm{~mm}$, and $2 \mathrm{~mm}$ models. Red lines denote the activity levels calculated by the BMS.

the morning peak of activity (Fig. 5A-C). When quantifying rest intensity, flies fed with a balanced diet showed no significant change as they aged (Figure $4 \mathrm{~A}-\mathrm{B})$. There may be a small increase in rest intensity between flies that are in the $15-25$ vs. 50-60 age groups, which is especially prominent in the dark period when rest is consolidated, but the difference is not statistically significant. This trend is similar to what was observed in D. melanogaster in which total sleep time increases with age, and the increase is most prominent earlier in life. It is possible that an increase sample size may increase the statistical significance of our observation. However, if A. ludens were fed with high sugar diet, their activity profiles show a much more apparent reduction in amplitude at old age (Figure 5D-F), perhaps reflecting premature decrease in mobility, energy level, and healthspan. Interestingly, the rest intensity of these flies in the dark period also showed a dramatic drop at old age (Figure 4D), indicating that perhaps rest in these flies may be decreased in quantity or less consolidated, also an indicator of a deterioration of health. Taken together, our results suggest that detrimental effect of high sugar or suboptimal diet may not manifest itself until the animal ages. Next, it will be interesting to determine the effects of diet on activity patterns in free-running conditions, e.g. constant darkness, to examine the influence of diet on circadian mechanisms that regulate activity patterns in $A$. ludens. Studies using D. melanogaster showed that older flies tend to have increasingly long period, until eventually activity patterns degenerate into arrhythmicity ${ }^{15}$. Using activity patterns of tephritid fruit fly as a model and our data presented here as a baseline, together with other behavioral parameters that BMS is capable of classifying, we can now perform studies to explore aging intervention ${ }^{26,29,30}$ in relation to diet. Moreover, we can explore the use of other behavioral parameters in addition to rest/activity patterns as biomarkers of aging and predictors of lifespan ${ }^{14}$.

In addition to providing a better understanding of the basic physiology of A. ludens, a serious insect pest of fruit crops and an insect model for aging and demographic studies, our study also reaffirmed that video-based high-resolution monitoring systems, such as BMS, provide a more accurate representation of insect behavior, even as simple and coarse as activity patterns. Monitoring systems with lesser resolution are more likely to overestimate rest or sleep, as we have clearly shown in this study. The capability of BMS, as shown previously ${ }^{26}$, is clearly not limited to differentiating between activity vs. inactivity. BMS is an automated video system capable of capturing, and more importantly classifying behaviors such as walking, resting, flying, feeding, drinking, and grooming. In the current version of BMS, there are two main drawbacks that accompany its increased resolution and capability. First, in order to record fine movements in microscopic scale for classifying a wide range of behaviors, high magnification cameras are necessary. This limits the recording field such that one camera cannot monitor all fly habitats simultaneously and has to rotate between different habitats, leading to non-continuous recordings, i.e. each fly is recorded for a 1-minute period every 18-20 minutes. Ongoing optimization of BMS to achieve continuous monitoring of all experimental animals includes the use of higher resolution recording cameras as well as the use of multiple cameras simultaneously. Continuous recording is necessary to accurately assess behavioral parameters, e.g. length of rest bouts. The second drawback, which is the requirement for increased computational 
Full Diet

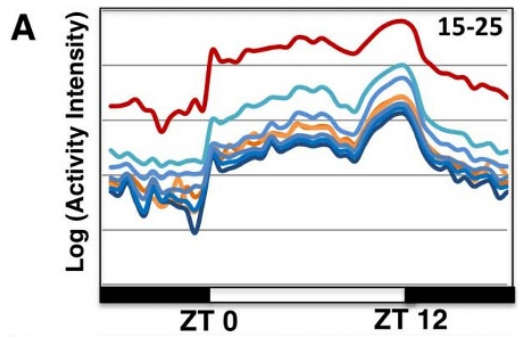

B

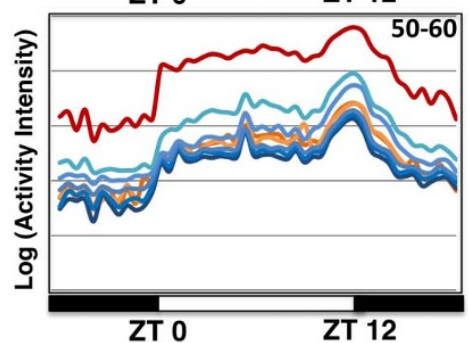

C

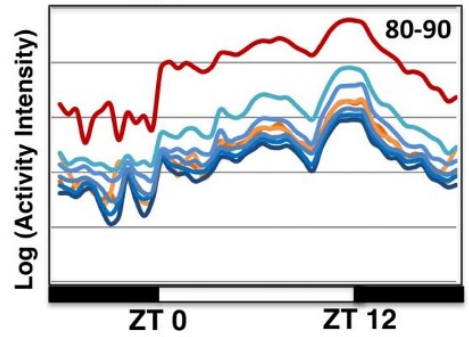

Sugar Diet

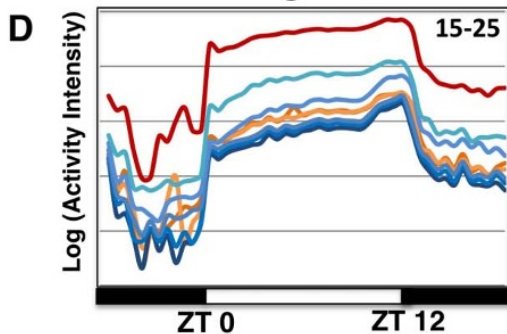

E

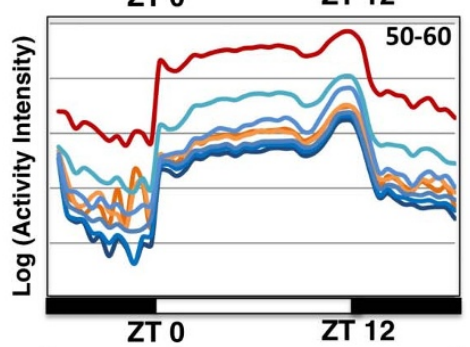

$\mathbf{F}$

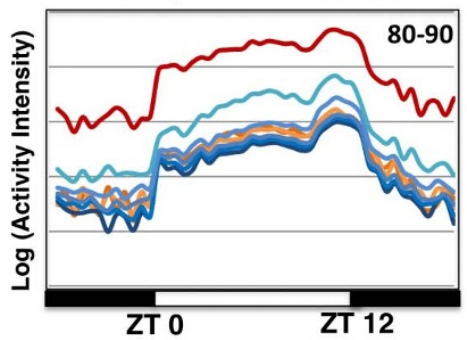

Figure $6 \mid$ Daily activity pattern by age groups as calculated using different activity monitoring models on logarithmic scale. Average diet-, age-, and model-specific activity patterns of $A$. ludens females through a 24-hour period. Each line represents the diurnal rest-activity patterns of $A$. ludens females averaged over eleven-day periods, ages 15-25, 50-60, and 80-90. (A-C) Females reared using full diet; (D-F) Females reared using sugar diet ( $\mathrm{n}=16$ ). Activity, plotted on the Y-axis, is on a logarithmic scale to show detail profile of each model. Dark bar below chart indicates the daily 12 -hour dark (lightsoff) period; white bar indicates the 12-hour light period. ZT0 indicates the time when lights turn on, and ZT12 indicates the time when lights turn off. Results from EBS (enclosure bisection simulation) X-axis and Y-axis bisections are represented by dark and light orange lines. Five blue-shaded lines from dark to light depict the results from DDS (distance displacement simulation) $20 \mathrm{~mm}, 15 \mathrm{~mm}, 10 \mathrm{~mm}, 5 \mathrm{~mm}$, and 2 mm models. Red lines denote the activity levels calculated by the BMS.

power for automation of behavioral classification and data storage capacity as compared to monitoring systems with less resolution, will be further magnified with the optimization of BMS to achieve continuous monitoring. However, with the fast pace of progress in computational technologies and parallel data processing, we anticipate that the time and cost to process such large behavioral bioinformatics data sets, not unlike those in the field of genomics, transcriptomics, and proteomics, will be dramatically reduced in a short time frame.

\section{Methods}

Fly husbandry and collection of data using behavioral monitoring system (BMS). Conditions for rearing of $A$. ludens and BMS experimental conditions were as previously described ${ }^{26}$. Full diet contained sugar and autolyzed yeast (MP

Biomedicals, cat. \#103304) at the ratio of 9:1, and was made by mixing 9 parts of sugar with 1 part of autolyzed yeast. Sugar diet contained sugar only. The experiments were conducted in environmentally controlled conditions where the temperature was kept at $25^{\circ} \mathrm{C}$; lights-on time was $7 \mathrm{am}$ and lights-off time was $7 \mathrm{pm}$. The dataset is a result of two replicate experiments yielding lifetime behavior patterns of 32 female flies, with 16 individuals receiving full diet and the other 16 receiving sugar-only diet. Fresh food and water were provided to flies weekly. Recording was initiated upon eclosion of flies, and lasted through the lifetime of the experimental animals. The sampling rate of the BMS was 5 images per second, and each BMS cage, containing one fly, was sampled for a consecutive duration of 60 seconds. This results in a total of 300 data points per bout of recording per fly. Due to the number of cages that required data sampling by a single video unit and required data processing time, each BMS was sampled every $18-20$ minutes.

Criteria used to define rest and activity in BMS, enclosure bisection simulation model and distance displacement simulation model. BMS allows the recreation of $3 \mathrm{D}$ trajectories of fly movement and uses that information to automate classification of six behaviors, including resting, micro-movement, walking, flying, feeding, and drinking ${ }^{26}$. The goal of this study was to analyze rest-activity cycle, and in-house scripts were generated to classify the BMS-recorded behavior to reflect rest vs. active only. Here we described the criteria used to define rest using (1) BMS, (2) the simulated enclosure bisection model, and (3) the simulated distance displacement model.

BMS: Currently, sleep periods in Drosophila melanogaster must be greater than 5 minutes in duration to be classified as sleep ${ }^{6}$. We are referring the inactivity assessed in our study as rest instead of sleep since there is currently no empirical evidence that the inactivity in A. ludens we are observing in our experiments represent bona-fide sleep as defined in $D$. melanogaster and mammals, e.g. whether it is accompanied by changes in arousal threshold ${ }^{11}$. To include a given 1-minute period as part of a rest bout, $90 \%$ or more of the behavior in that 1-minute (300-data points) recording session must be rest as defined by $\mathrm{BMS}^{26}$. The $10 \%$ active behavior limit only allows micro-movement behavior (twisting, rotating, flexing wings). If the fly moved more than $2 \mathrm{~mm}$ in the $\mathrm{X}$, Y, or Z plane (xMax - xMin $>2 \mathrm{~mm}$ ) during a 1-minute (300points) recording session, the 1-minute recording was not counted as rest behavior. Furthermore, movement must be less than $2 \mathrm{~mm}$ in $\mathrm{X}, \mathrm{Y}$, and $\mathrm{Z}$ directions between two 1-minute recordings (which spans on average 18-20 minutes due to the nature of BMS recordings) in order for those recordings to be included in a rest bout. In other words, if the fly moves more than $2 \mathrm{~mm}$ from its previous location in the 18-20 minutes when BMS is monitoring other flies, then the fly was considered to have stopped resting and rest duration was reset again to zero.

Simulated enclosure bisection and distance displacement models: When a fly crossed the threshold criteria for the enclosure bisection model or threshold distance set for the distance displacement model less than 5 minutes after it previously crossed that same distance, the simulation models assumed the fly had not rest. Every time the fly crossed the threshold distance, the rest duration was reset to zero. The smallest quiescence period in this data is $>10$ minutes due to the BMS monitoring cycle. The difference between the different simulation models is as follows: For the enclosure bisection simulation model, the fly must remain on one side of the cage to achieve quiescence. Crossing the midpoint of the given axis (X or Y) of the cage 'breaks the beam' (Figure 1A) and resets the rest duration value to zero. For the distance displacement simulation models, the fly must remain within the given distance $(2 \mathrm{~mm}$, $5 \mathrm{~mm}, 10 \mathrm{~mm}, 15 \mathrm{~mm}$, and $20 \mathrm{~mm}$ ) from an initial position to achieve quiescence. 
Moving beyond the given radius from its initial position crosses the threshold limit, and thus sets a new initial position, resetting the rest duration value to zero. The greater the radial distance in the different displacement models (e.g. $2 \mathrm{~mm}$ vs. $20 \mathrm{~mm}$ ), the more movement afforded the fly while still being considered resting. Therefore, the $2 \mathrm{~mm}$ displacement model should more accurately measure actual behavior and most similar to BMS. The difference in activity levels as calculated using the different models were assessed using Student's t-test. A value of $p<0.05$ was considered as statistically significant.

1. Edwards, B. A. et al. Aging and sleep: physiology and pathophysiology. Semin Respir. Crit. Care Med. 5, 618-633 (2010).

2. Iliadi, K. G. \& Boulainne, G. L. Age-related behavioral changes in Drosophila. Annals of the NY Acad. Sci. 1197, 9-18 (2010).

3. Ohayon, M. M., Carskadon, M. A., Guilleminault, C. \& Vitiello, M. V. Metaanalysis of quantitative sleep parameters from childhood to old age in healthy individuals: developing normative sleep values across the human lifespan. Sleep 27, 1255-1273 (2004).

4. Bushey, D., Hughes, K. A., Tononi, G. \& Cirelli, C. Sleep, aging, and lifespan in Drosophila. BMC Neuroscience 11, 56 (2010).

5. Cirelli, C. Searching for sleep mutants of Drosophila melanogaster. Bioessays 25 940-949 (2003).

6. Hendricks, J. C. et al. Rest in Drosophila is a sleep-like state. Neuron 25, 129-138 (2000).

7. Ho, K. S. \& Sehgal, A. Drosophila melanogaster: an insect model for fundamental studies of sleep. Methods Enzymol. 393, 772-793 (2005).

8. Koh, K., Evans, J. M., Hendricks, J. C. \& Sehgal, A. A Drosophila model for ageassociated changes in sleep:wake cycles. Proc. Natl. Acad. Sci. USA 103, 13843-13847 (2006).

9. Shaw, P. J., Cirelli, C., Greenspan, R. J. \& Tononi, G. Correlates of sleep and waking in Drosophila melanogaster. Science 287, 1834-1837 (2000).

10. Shaw, P. Awakening to the behavioral analysis of sleep in Drosophila. J. Biol. Rhythms 18, 4-11 (2003).

11. Huber, R. et al. Sleep homeostasis in Drosophila melanogaster. Sleep 27 (4), 628-639 (2004).

12. Cirelli, C. et al. Reduced sleep in Drosophila Shaker mutants. Nature 434 (7037), 1087-1092 (2005).

13. Bushey, D., Huber, R., Tononi, G. \& Cirelli, C. Drosophila Hyperkinetic mutants have reduced sleep and impaired memory. J. Neurosci. 27 (20), 5384-5393 (2007).

14. Koudounas, S., Green, E. W. \& Clancy, C. Reliability and variability of sleep and activity as biomarkers of ageing in Drosophila. Biogerontology 13,489-499 (2012).

15. Luo, W. et al. Old flies have a robust central oscillator but weaker behavioral rhythms that can be improved by genetic and environmental manipulations. Aging Cell 11, 428-438 (2012).

16. Rosato, E. \& Kyriacou, C. P. Analysis of locomotor activity rhythms in Drosophila. Nature Protocols 1, 559-568 (2006)

17. Zordan, M. A., Benna, C. \& Mazzotta, G. Monitoring and analyzing Drosophila circadian locomotor activity. In Circadian Rhythms Methods and Protocols (ed. Rosato, E.) Methods in Molecular Biology series (Humana, Totowa, New Jersey, 2007).

18. Chiu, J. C., Low, K. H., Pike, D., Yildrim, E. \& Edery, I. Assaying locomotor activity to study circadian and sleep parameters in Drosophila.J Vis. Exp. 43, doi: 10.3791/ 2157 (2010).

19. Rund, S. S., Hou, T. Y., Ward, S. M., Collins, F. H. \& Duffield, G. E. Genome-wide profiling of diel and circadian gene expression in the malaria vector Anopheles gambiae. Proc. Natl. Acad. Sci. USA 108, E421-430 (2011).

20. Martin, J. R. A potrait of locomotor behavior in Drosophila determined by videotracking paradigm. Behavioural Processes 67, 207-219 (2004).

21. Valente, D., Goani, I. \& Mitra, P. P. Analysis of the trajectory of Drosophila melanogaster in a circular open field arena. PLoS One 2 (10), 21083 (2007).

22. Zimmerman, J. E., Raizen, D. M., Maycock, M. H., Maislin, G. \& Pack, A. I. A video method to study Drosophila sleep. Sleep 31, 1587-1598 (2008).

23. Branson, K., Robie, A. A., Bender, J., Perona, P. \& Dickinson, M. H. High throughput ethomics in large groups of Drosophila. Nature Methods 6, 451-457 (2009).

24. Donelson, N. et al. High-resolution positional tracking for long-term analysis of Drosophila sleep and locomotion using the "Tracker" program. PLoS One 7 (5), e37250 (2012).
25. Gilestro, G. F. Video tacking and analysis of sleep in Drosophila melanogaster. Nat. Prot. 7 (5), 995-1007 (2012).

26. Zou, S. et al. Recording lifetime behavior and movement in an invertebrate model. PLoS One 6 (4), e18151 (2011).

27. Peuhkuri, K., Sihvola, N. \& Korpela, R. Diet promotes sleep duration and quality. Nutr. Res. 32, 309-319 (2012)

28. Carey, J. R. et al. Biodemography of a long-lived tephritid: Reproduction and longevity in a large cohort of female Mexican fruit flies, Anastrepha ludens. Experimental Gerontology 40, 793-800 (2005).

29. Carey, J. R. et al. Longevity-fertility trade-offs in the tephritid fruit fly Anastrepha ludens, across dietary-restriction gradients. Aging Cell 7, 470-477 (2008).

30. Zou, S. et al. The prolongevity effect of resveratrol depends on dietary composition and calorie intake in a tephritid fruit fly. Exp Gerontol. 44, 472-476 (2009).

31. Zou, S. et al. Prolongevity effects of an oregano and cranberry extract are diet dependent in the Mexican fruit fly (Anastrepha ludens). J Gerontol A Biol Sci 65, 41-50 (2010).

32. Aluja, M. Bionomics and management of Anastrepha. Annu. Rev. Entomol. 38, 155-178 (1994)

33. NAPPO. Anastrepha ludens (Mexican fruit fly) eradicated in the United States. Phytosanitary Alert System. http://www. pestalert.org/oprDetail.cfm?oprID = 511 (January 18 2012).

34. Yamazaki, M. et al. High calorie diet augments age-associated sleep impairment in Drosophila. Biochem. Biophys. Res. Commun. 417, 812-816 (2012).

35. Stoleru, D., Peng, Y., Agosto, J. \& Rosbash, M. Coupled oscillators control morning and evening locomotor behaviour of Drosophila. Nature 431 (7010), 862-868 (2004).

36. Grima, B., Chelot, E., Xia, R. \& Rouyer, F. Morning and evening peaks of activity rely on different clock neurons of the Drosophila brain. Nature 431 (7010), 869-873 (2004)

37. Currie, J., Goda, T. \& Wijnen, H. Selective entrainment of the Drosophila circadian clock to daily gradients in environmental temperature. BMC Biol. 7, 49 (2009).

38. Vanin, S. et al. Unexpected features of Drosophila circadian behavioural rhythms under natural conditions. Nature 484 (7394), 371-375 (2012).

39. Aluja, M., Pinero, J., Jacome, I., Diaz-Fleischer, F. \& Sivinski, J. Behavior of flies in the Genus Anastrepha in Fruit Flies (Tephritidae): Phylogeny and evolution of behavior, Aluja, M. and Norrbom, A. L. (eds.). CRC Press, New York NY, USA (2000).

\section{Acknowledgements}

We thank A. Oropeza, R. Bustamente, E. de Leon, S. Rodriguez, R. Rincon and G. Rodas for excellent technical support and G. Olivera-Romero, S. Soriano-Monje and D. Tenorio-Pérez for assistance in the development of the BMS at INAOE. The Moscamed-Moscafrut facility (DGSV, SENASICA) in Metapa, Chiapas, Mexico, for providing mexflies and lab space. This work was supported by NIH P01 AG022500-01 and P01 AG08761-10 to J.R.C.; the Intramural Research Program of NIA, NIH to S.Z.; and NIH NS069542 to J.C.C.

\section{Author contributions}

Conceived and designed the experiments: J.R.C., S.Z., P.L., L.A.-R., D.K.I. Performed the experiments: S.Z., P.L. Analyzed the data: J.C.C., K.K., and J.R.C. Wrote the paper: J.C.C., K.K. and J.R.C.

\section{Additional information}

Competing financial interests: The authors declare no competing financial interests.

License: This work is licensed under a Creative Commons

Attribution-NonCommercial-NoDerivs 3.0 Unported License. To view a copy of this license, visit http://creativecommons.org/licenses/by-nc-nd/3.0/

How to cite this article: Chiu, J.C. et al. Deleterious effect of suboptimal diet on rest-activity cycle in Anastrepha ludens manifests itself with age. Sci. Rep. 3, 1773; DOI:10.1038/ srep01773 (2013). 


\section{SCIENTIFIC \\ REPRTS}

DOI: 10.1038/srep02450

SUBJECT AREAS: AGEING

EVOLUTION

ECOLOGY

BEHAVIOURAL ECOLOGY

SCIENTIFIC REPORTS:

$3: 1773$

DOI: $10.1038 /$ srep0 1773

(2013)

Published:

3 May 2013

Updated:

14 August 2013
A

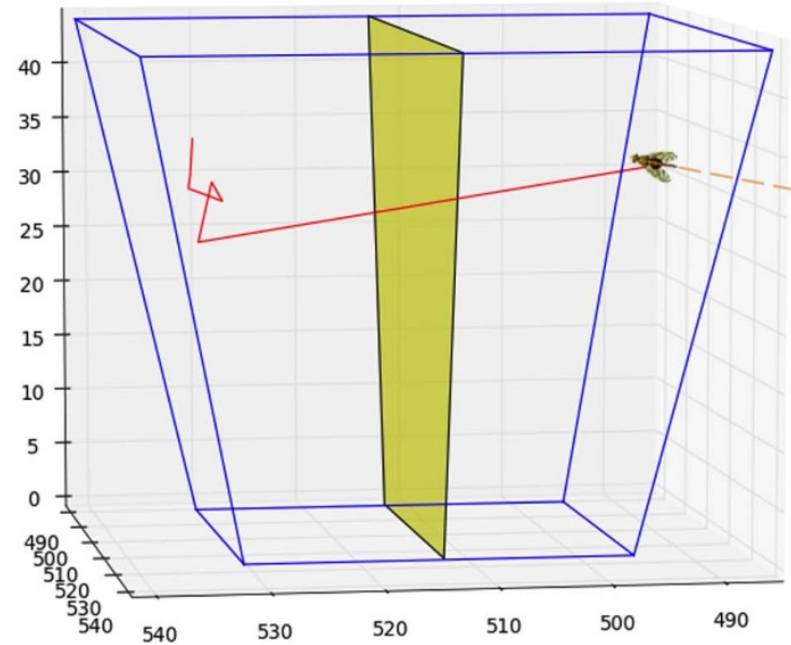

B

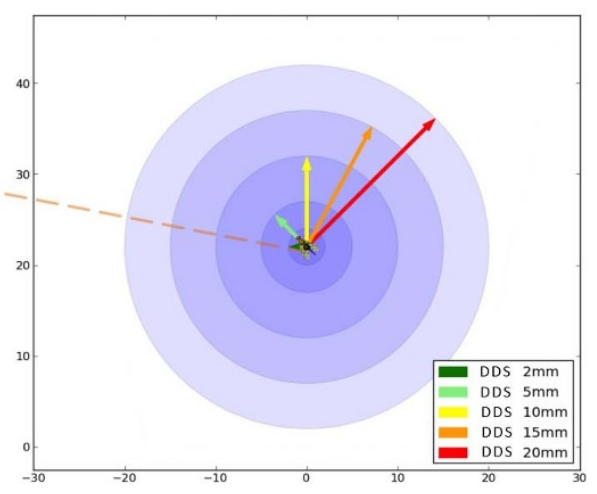

